\title{
Flow-Mediated Interactions between Two Self-Propelled Flapping Filaments in Tandem Configuration
}

\author{
Xiaojue Zhu, ${ }^{\dagger}$ Guowei He, and Xing Zhang \\ The State Key Laboratory of Nonlinear Mechanics (LNM), Institute of Mechanics, Chinese Academy of Sciences, Beijing 100190, China
} (Received 15 April 2014; revised manuscript received 20 August 2014; published 3 December 2014)

The mechanism by which aggregates of active swimmers are formed is an intriguing problem. In this Letter we show that, for two tandem self-propelled filaments driven by harmonic plunging motions of identical frequency and amplitude, stable configurations can be spontaneously formed by locking the trajectories onto the vortex centers. Further analysis indicates that the grouping energetics is also dictated by the wake vortex structure. The rationale behind the energetic advantage of the follower is found to be the interplay among actuation, self-propulsion, and the vortical fluid environment.

DOI: 10.1103/PhysRevLett.113.238105

PACS numbers: 47.63.M-, 46.70.-p, 47.32.-y

The aggregates of moving bodies in fluid are ubiquitous in nature. Some well-known examples are sedimenting particles and fibers, racing bicyclists, flying birds, and swimming fish. Here we distinguish an actively moving body from a passively moving body. The forward motion of the former is due to self-propulsion (interacting with the fluid), whereas the forward motion of the latter is directly caused by external forces (such as gravity or towing).

For passively moving bodies in tandem configuration, the flow-mediated interactions are often rationalized by the conventional hydrodynamic drafting (in which the follower enjoys drag reduction) [1]. However, "inverted" hydrodynamic drafting (where the leader enjoys drag reduction) was found in some recent studies on tandem deformable bodies [2-6]. As pointed out in Ref. [2], the tandem configuration of two passively moving bodies is unstable due to the unequal drag forces.

The interactions among actively moving bodies in fluid are more complicated. Although numerous studies on fish schools [7], bird flocks [8], and systems containing bacteria or active particles $[9,10]$ can be found in the literature, the mechanism by which stable aggregations are formed remains unclear. The hydrodynamic characteristics of two coupled flapping (or traveling wavy) foils have been studied in some previous works [11-13]. For fish schools, Weihs predicted that a diamondlike configuration of the positions of individuals is energetically optimal [14]. However, there is little biological evidence that such configuration occurs in nature [15]. In a more recent study, several different configurations (line, phalanx, diamond, and rectangular) are all found to be hydrodynamically advantageous [16]. Thus, the function of hydrodynamics and the optimal configuration in fish schools are still controversial.

In this Letter, we consider the coupling of two tandem active swimmers through their shared fluid environment. Our aim here is to demonstrate that (a) a stable configuration can be formed spontaneously with the help of the vortex street behind the leader and (b) the energetics of this configuration is also dictated by the vortex structure of the wake.

To better represent the real-world biolocomotion, we propose to include both actuation and self-propulsion in the present model. This is very different from the model used in some previous works $[11,12,14]$, where the body was held fixed in an oncoming flow. Although a single actively flapping body in self-propulsion has been studied before [17-21], the multibody interactions under such condition have never been considered.

A sketch of the present model is shown in Fig. 1. Two flapping filaments are driven at the leading edge by harmonic plunging motions and are free to move leftwards or rightwards, depending on the hydrodynamic force. The filaments are inextensible and the surrounding flow is two dimensional, incompressible, and laminar. The motions of the fluid and the filaments are governed by the Navier-Stokes equations coupled with the geometrically non-linear structural equation [20-22], written in a dimensionless form as

$$
\begin{aligned}
& \frac{\partial \mathbf{u}}{\partial t}+(\mathbf{u} \cdot \nabla) \mathbf{u}=-\nabla p+\frac{1}{\operatorname{R} e_{f}} \nabla^{2} \mathbf{u}+\mathbf{f}, \\
& \nabla \cdot \mathbf{u}=0, \\
& \beta \frac{\partial^{2} \mathbf{X}}{\partial t^{2}}-\frac{\partial}{\partial s}\left(\zeta(s) \frac{\partial \mathbf{X}}{\partial s}\right)+\frac{\partial^{2}}{\partial s^{2}}\left(\gamma \frac{\partial^{2} \mathbf{X}}{\partial s^{2}}\right)=-\mathbf{F}, \\
& \frac{\partial \mathbf{X}}{\partial s} \cdot \frac{\partial \mathbf{X}}{\partial s}=1 .
\end{aligned}
$$

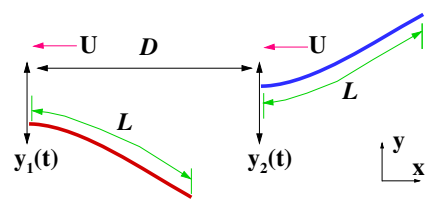

FIG. 1 (color online). Two self-propelled tandem filaments driven by plunging motions. The gap distance between the two filaments is defined as $G=D-L$. 
Here, $\mathbf{u}, p$, and $\mathbf{X}=\left(X^{(1)}, X^{(2)}\right)$ are the fluid velocity, fluid pressure, and displacement of the filaments, respectively. $\mathbf{f}$ and $\mathbf{F}$ are the Eulerian and Lagrangian force densities which represent the interactions between the fluid and the filaments.

The dimensionless governing equations above are obtained by scaling space and time with $L$ and $L / U_{\text {ref }}$, respectively. Here, $L$ is the length of the filament and $U_{\text {ref }}$ is the reference velocity. The plunging motions at the two leading edges are prescribed as $y_{1}=A \cos (2 \pi f t)$ and $y_{2}=A \cos (2 \pi f t+\Delta \phi)$, respectively, with $\Delta \phi$ being the phase difference. The reference velocity used in the scaling is the maximum flapping velocity of the plunging motion, i.e., $U_{\text {ref }}=2 \pi A f$.

Five dimensionless parameters arise in the scaling, i.e.,

$$
\begin{aligned}
\bar{A} & =A / L, \\
\operatorname{Re}_{f} & =\frac{\rho_{f} U_{\mathrm{ref}} L}{\mu}, \quad \beta=\frac{\rho_{s}}{\rho_{f} L}, \\
\gamma & =\frac{B}{\rho_{f} U_{\mathrm{ref}}^{2} L^{3}}, \quad \zeta(s)=\frac{T(s)}{\rho_{f} U_{\mathrm{ref}}^{2} L},
\end{aligned}
$$

which are the dimensionless amplitude, Reynolds number, mass ratio, dimensionless bending rigidity, and dimensionless tension, respectively. $\rho_{s}, T(s)$, and $B$ are the linear density, tension, and bending rigidity of the filament, respectively. $\rho_{f}$ and $\mu$ are the area density and dynamic viscosity of the fluid, respectively. Here, the variable tension $T(s)$, which is used to enforce the inextensibility condition, satisfies Poisson's equation [22].

The governing equations are solved by using the immersed boundary method. The values of physical parameters used in the simulations are listed in Table I. For the sake of generality, four representative cases have been selected here. Two of them are rigid cases and two of them are flexible ones. Three of them are of the $2 S$ wake mode and one of them is of the $2 P$ wake mode. (For the details regarding the numerical method and the selection of parameter values, please see Ref. [23].) For reference

TABLE I. Values of control parameters used in simulations. The two bending rigidities in $1 \mathrm{~A}$ and $1 \mathrm{~B}$ are the optimal ones for achieving the fastest forward speed. For $1 \mathrm{~B}$ and $2 \mathrm{~B}$, the two filaments are actually rigid due to the extremely large values of bending rigidities. $1 \mathrm{~B}, 2 \mathrm{~A}$, and $2 \mathrm{~B}$ are of the $2 S$ wake mode (in which one vortex is shed within half-cycle), whereas $1 \mathrm{~A}$ is of the $2 P$ wake mode (in which two vortices with the same sign are shed within half-cycle).

\begin{tabular}{lcccc}
\hline \hline Case & $\operatorname{Re}_{f}$ & $\bar{A}$ & $\beta$ & $\gamma$ \\
\hline $1 \mathrm{~A}$ & 200 & 0.50 & 0.2 & 0.8 \\
$1 \mathrm{~B}$ & 200 & 0.50 & 0.2 & $10^{4}$ \\
2A & 200 & 0.20 & 0.2 & 3.0 \\
2B & 200 & 0.20 & 0.2 & $10^{4}$ \\
\hline \hline
\end{tabular}

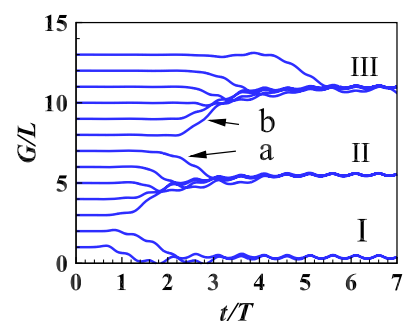

(a)

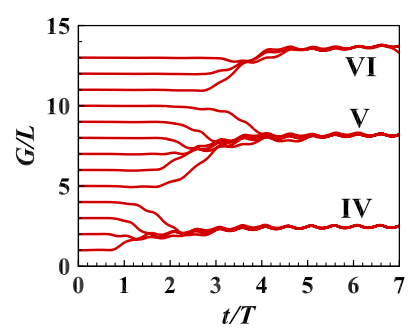

(b)
FIG. 2 (color online). Separation distance as a function of time for case $1 \mathrm{~A}$. (a) In-phase scenario $(\Delta \phi=0)$, (b) antiphase scenario $(\Delta \phi=\pi)$. For the purpose of indexing, the equilibrium distances achieved are labeled as roman numerals while two evolutionary paths are labeled as "a" and "b," respectively.

purposes, in each representative case, one filament in isolated swimming is also simulated.

To identify the possible formation of a stable configuration, in Fig. 2 we show the evolution of separation distance with time for case $1 \mathrm{~A}$. It is seen that for both the inphase $(\Delta \phi=0)$ and the antiphase $(\Delta \phi=\pi)$ scenarios, an equilibrium separation distance can be achieved within 3-6 periods from the start. Another interesting finding is that for all initial separation distances studied here, only discrete values of equilibrium distances are realizable (which are labeled as I-VI in Fig. 2).

Further investigation indicates that the stable configurations achieved can be categorized into two subtypes: compact configuration and regular configuration. The wake structure corresponding to the compact configuration for case 1A (labeled as I in Fig. 2) is shown in Fig. 3(a). Because of the narrow gap spacing between them, the two individuals behave like one single and longer filament and the reversed Karman vortex street is only seen behind the rear one. Figures 3(b) and Fig. 3(c) are the wake structures corresponding to regular configurations for case 1A (labeled as II and IV, respectively, in Fig. 2). One salient feature of this configuration is that the reversed Karman vortex street in between two filaments looks just like the

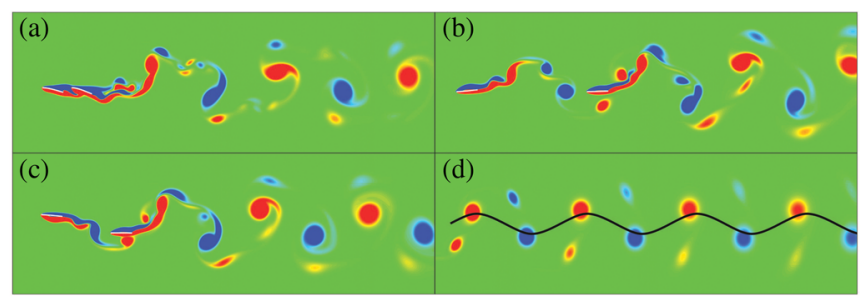

FIG. 3 (color online). Wake structures for different equilibrium separation distances in case 1A: (a) $G_{\text {eq }}=0.2 L$ (compact configuration), (b) $G_{\mathrm{eq}}=5.0 \mathrm{~L}$ (regular configuration), (c) $G_{\text {eq }}=2.0 \mathrm{~L}$ (regular configuration), (d) superimposition of rear filament's leading-edge trajectory for (b) on vortex street generated by one filament in isolated swimming. Red and blue colors denote positive (anticlockwise) and negative (clockwise) vorticity, respectively. 
one behind an isolated swimmer. This indicates that the influence of the flow-mediated interactions on the leader is minor. The strong influence of the flow-mediated interactions on the follower can be seen from the complex vortical structures in the wake of the follower (which are the "footprints" of vortex-vortex and vortex-body interactions). Another noteworthy feature in regular configurations is that the equilibrium gap distance is correlated with the streamwise spatial period of the reversed Karman vortex street. This "vortex locking" behavior can be more clearly visualized by superimposing the trajectory of the leading edge of the follower in such a configuration onto the vortex street generated by an isolated swimmer which is started exactly the same as that of the "leader" [see Fig. 3(d)]. For regular configurations, it is also confirmed that the vortex locking behavior and the shape of the trajectory [as that depicted in Fig. 3(d)] are not affected by the phase difference between the two filaments, although the stable separation distance changes continuously with the variation of $\Delta \phi$ (see Ref. [23] for further explanation).

By examining the regular configurations achieved (see videos in Ref. [23]), it is seen that the trajectories of the rear filaments are closely associated with the wake vortex structure. In the wake of the $2 S$ mode (such as that in case $2 \mathrm{~A}, 1 \mathrm{~B}$, and $2 \mathrm{~B}$ ), the follower swims through the cores of positive and negative vortices alternatively. In the wake of the $2 P$ mode (such as that in case 1A), the follower will always swim through the cores of the strongest vortices within each vortex pair. A hypothesized mechanism for the behavior of the rear filament in the present work is shown in Fig. 4. We found that swimming through the vortices in the reversed Karman vortex street is the optimal trajectory for the follower, in the sense of obtaining energetic benefits, since it requires moving with the local lateral flow rather than moving against it. Previously, swimmers have been hypothesized to slalom between the vortex cores in the wake of a rigid or flexible object (facing the Karman vortex street) $[24,25]$. The argument above can also be used to rationalize such phenomena.

To further demonstrate the process of the formation of a stable configuration, the time histories of the instantaneous

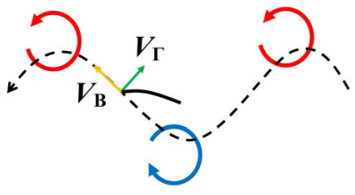

(a)

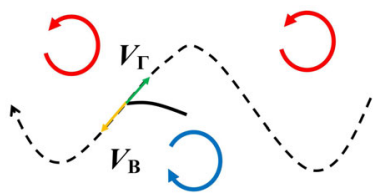

(b)
FIG. 4 (color online). Comparison of two possible trajectories adopted by the rear filament in a regular configuration. (a) By swimming through the vortex cores, the rear filament moves with the local lateral flow. (b) By slaloming between the vortex cores, the rear filament moves against the local lateral flow. The positive and negative vortices are denoted by red and blue circular arrows, respectively. $\mathbf{V}_{\Gamma}$ is the velocity induced by the vortex dipole. $\mathbf{V}_{B}$ is the velocity of the leading edge of the rear filament. forward speeds in the front and rear filament are shown in Fig. 5. The evolution of the speed in an isolated swimmer is also plotted for reference purposes. It is seen that the time history of the speed for the front filament is exactly the same as that for an isolated filament. The evolution of the speed in the rear filament can be divided into three stages. In the first stage, the rear filament has not encountered the vortices shed from the front one. Thus, the time histories of the two speeds are exactly the same. In the second stage, the rear filament undergoes a sudden acceleration (or deceleration) process. In the third stage, the speed of the rear filament undergoes an overshooting (or undershooting) process before it is finally relaxed to the equilibrium value. In some phenomenological models for the description of fish schooling, repulsive and attractive forces were combined to yield an equilibrium separating distance between members [26]. Based on the results of this work, the sources of these hypothesized forces can be attributed to the vortex-body interactions.

We now look at the effects of flow-mediated interactions on two important indicators for quantifying the propulsive performance, namely, the cruising speed and the swimming power. The cruising speed is defined as the averaged forward speed at the equilibrium state, i.e.,

$$
\bar{U}_{c}=-\frac{1}{T} \int_{0}^{T}\left(\left.\frac{\partial X^{(1)}}{\partial t}\right|_{s=0}\right) d t
$$

where $T$ is the flapping period. The swimming power $P$ is defined as the average input power that is required to produce the oscillation and the forward movement, i.e.,

$$
\bar{P}=\frac{1}{T} \int_{0}^{T} P(t) d t=\frac{1}{T} \int_{0}^{T}\left[\int_{0}^{1}\left(\mathbf{F} \cdot \frac{\partial \mathbf{X}}{\partial t}\right) d s\right] d t .
$$

The cruising speed and swimming power of the two filaments in stable configurations are listed in Table II. The

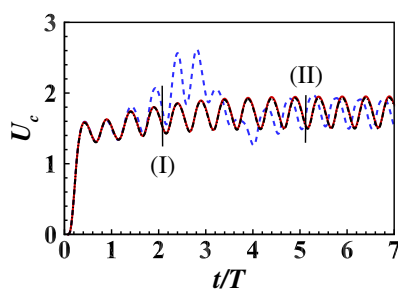

(a)

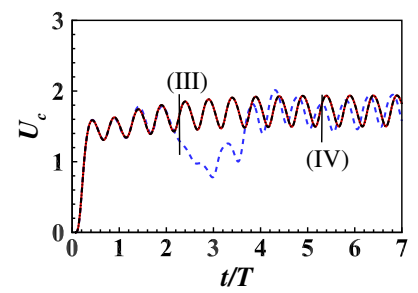

(b)
FIG. 5 (color online). The streamwise velocity of the leading edge as a function time for case $1 \mathrm{~A}$ (in-phase scenario). (a) $G_{0}=7 L$ [labeled as "a" in Fig. 2(a)], (b) $G_{0}=8 L$ [labeled as "b" in Fig. 2(a)]. The solid and dashed lines denote the forward velocities of the leading edges of the front and rear filaments, respectively. The dash-dotted lines denote the velocity of a single filament in isolated swimming. [The wake structures at four time instants (I, II, III, and IV) and the videos for the evolution of wake structures are given in the Supplemental Material [23].] 
TABLE II. Cruising speed and swimming power for different cases. The superscript (i) denotes one filament in isolated swimming, superscripts $(c)$ and $(r)$ denote compact configuration and regular configuration, respectively, superscripts $(u)$ and $(d)$ denote upstream and downstream filament, respectively.

\begin{tabular}{lccccc}
\hline \hline Case & $\bar{U}_{c}{ }^{(u)}$ & $\bar{U}_{c}{ }^{(d)}$ & $\bar{P}^{(u)}$ & $\bar{P}^{(d)}$ & Mode \\
\hline $1 \mathrm{~A}^{(i)}$ & 1.74 & $\ldots$ & 1.22 & $\ldots$ & $2 P$ \\
$1 \mathrm{~A}^{(c)}$ & 2.08 & 2.08 & 1.39 & 1.39 & $2 P$ \\
$1 \mathrm{~A}^{(r)}$ & 1.74 & 1.74 & 1.22 & 1.23 & $2 P$ \\
$1 \mathrm{~B}^{(i)}$ & 1.29 & $\ldots$ & 1.22 & $\ldots$ & $2 S$ \\
$1 \mathrm{~B}^{(r)}$ & 1.29 & 1.29 & 1.22 & 1.02 & $2 S$ \\
$2 \mathrm{~A}^{(i)}$ & 2.03 & $\ldots$ & 2.54 & $\ldots$ & $2 S$ \\
$2 \mathrm{~A}^{(r)}$ & 2.03 & 2.03 & 2.54 & 2.02 & $2 S$ \\
$2 \mathrm{~B}^{(i)}$ & 1.17 & $\ldots$ & 1.78 & $\ldots$ & $2 S$ \\
$2 \mathrm{~B}^{(r)}$ & 1.17 & 1.17 & 1.78 & 1.36 & $2 S$ \\
\hline \hline
\end{tabular}

values for a filament in isolated swimming are also presented for comparison. Some important observations are as follows. In compact configurations, the filaments swim faster than an isolated swimmer but also consume more power. In this type of configuration, no energetic advantage of the follower is found. In regular configurations, the cruising speeds of the two filaments are the same as that of an isolated swimmer. The leader in this type of configuration always consumes the same power as that in an isolated swimmer. Energetic advantage of the follower is found in cases $1 \mathrm{~B}, 2 \mathrm{~A}$, and $2 \mathrm{~B}$, where the power consumption in the follower is around $80 \%$ of that in the leader. This provides indirect evidence that the follower is capable of exploiting fluid energy by interacting with the vortical wake. In case $1 \mathrm{~A}$, however, no energetic advantage is found in the follower (swimming powers in both filaments are almost equivalent). For this active system and within the scope of parameter values explored, the inverted drafting (energetic advantage of the leader) reported in Ref. [2] is never observed. For the regular configurations, we also found that the results above are not sensitive to the equilibrium distance achieved (within the range of this study). This phenomenon can be rationalized by the slow vortex decaying rate at this Reynolds number.

Here, we stress that the energetic advantage of the follower in some cases (those with $2 S$ wakes) cannot be explained by the conventional hydrodynamic drafting. The energetics advantage of the follower in the tandem configuration is also inconsistent with the prediction made by the theory of Weihs [14]. According to this theory, being straight behind another individual is a disadvantageous position for swimming (since here the swimmer is facing a backward jet induced by the reversed Karman vortices). This inconsistency is caused by the overly simplified assumption in Weihs's model, where actuation and selfpropulsion of the follower are not taken into account. From the hypothesized mechanism of energy saving in the follower depicted in Fig. 4(a), we can see that when the actuation is taken into consideration, the $y$ component of velocity is more important than the $x$ component. This is further confirmed by the fact that the contribution from the $y$ components of force and velocity is always dominant in the swimming power, while the contribution from the $x$ components is negligible. We can also see things from another perspective. The $y$ component of velocity is more important, because the only force doing work on the swimmer (the point force applied to the head) is in the $y$ direction.

The reason why no clear benefit is gained by the follower in the cases of $2 P$ wakes is presented as follows. From Fig. 4(a), it is seen that how much the follower can benefit from the wake depends on the $y$ component of the dipoleinduced velocity. In Fig. 6, the dipole-induced velocity of the $2 P$ case is compared with that of the $2 S$ case. For the $2 P$ case, only the velocity induced by the "stronger" dipole is considered since the velocity induced by the "weaker" dipole is rather small. By using the vortex-dipole model [27], the component of the dipole-induced velocity can be approximated as $V_{\Gamma}^{(2)}=(\Gamma / 2 \pi \xi) \sin \alpha$, where $\Gamma$ is the vortex circulation, $\xi$ is the distance between the two vortex centers, and $\alpha$ is the orientation angle between the $x$ direction and the direction of the dipole. No clear energetic benefit of the follower in the $2 P$ wakes can be explained by fact that the $y$ component of the dipole-induced velocity is too small (which is only about one eighth of the value for the $2 S$ case). The reduction of $V_{\Gamma}^{(2)}$ in the $2 P$ case is caused by the following factors: (a) weaker dipole strength, (b) longer distance between the two vortex centers, and (c) smaller angle between the $x$ direction and the direction of the dipole. (The values of $V_{\Gamma}^{(2)}, \Gamma, \xi$, and $\alpha$ are given in Ref. [23]).

In summary, we studied the flow-mediated interactions between two tandem self-propelled filaments. We showed that a stable configuration can be spontaneously formed with the help of the vortex street behind the leader. For both the rigid and the flexible filaments, energetic advantage of the leader was never observed. Whether the follower can enjoy energetic benefits was found to be dictated by the wake vortex structure. Although the geometric shape, the actuation, and the grouping configuration are highly simplified, the model problem studied here is still essential

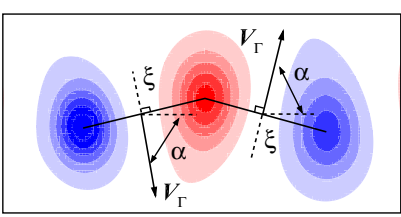

(a)

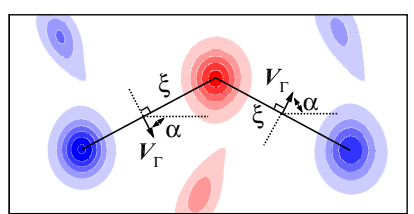

(b)
FIG. 6 (color online). A schematic diagram of the vortex-induce velocities (magnitude and orientation) in the wakes of (a) $2 S$ mode (case 2A) and (b) $2 P$ mode (case 1A). 
for understanding the coordinated collective behaviors in macroscopic swimmers.

This work is supported by Chinese Academy of Sciences (under Projects No. KJCX-SW-L08 and No. KJCX3-SYWS01), NSFC (under Project No. 11021262, No. 11023001, No. 11232011, and No. 11372331), and the National Basic Research Program of China (under Project No. 2013CB834100). We thank Detlef Lohse, Devaraj van der Meer, and Chao Sun at University of Twente for helpful discussions.

* Corresponding author.

zhangx@1nm.imech.ac.cn

†Present address: Physics of Fluids Group, University of Twente, 7500 AE Enschede, The Netherlands.

[1] M. M. Zdravkovich, J. Fluids Eng. 99, 618 (1977).

[2] L. Ristroph and J. Zhang, Phys. Rev. Lett. 101, 194502 (2008).

[3] S. Alben, J. Fluid Mech. 641, 489 (2009).

[4] L. Zhu, J. Fluid Mech. 635, 455 (2009).

[5] S. Kim, W. X. Huang, and H. J. Sung, J. Fluid Mech. 661, 511 (2010).

[6] E. Uddin, W. X. Huang, and H. J. Sung, J. Fluid Mech. 729, 563 (2013).

[7] F. E. Fish, Comments Theor. Biol. 5, 283 (1999).

[8] C. K. Hemelrijk and H. Hildenbrandt, Interface Focus 2, 726 (2012).

[9] C. Dombrowski, L. Cisneros, S. Chatkaew, R. E. Goldstein, and J. O. Kessler, Phys. Rev. Lett. 93, 098103 (2004).

[10] D. Saintillan and M. J. Shelley, Phys. Rev. Lett. 100, 178103 (2008).
[11] G. V. Lauder, E. J. Anderson, J. Tangorra, and P. G. A. Madden, J. Exp. Biol. 210, 2767 (2007).

[12] G. J. Dong and X. Y. Lu, Phys. Fluids 19, 057107 (2007).

[13] Z. J. Wang and D. Russell, Phys. Rev. Lett. 99, 148101 (2007).

[14] D. Weihs, Nature (London) 241, 290 (1973).

[15] B. L. Partridge and T. J. Pitcher, Nature (London) 279, 418 (1979).

[16] C. K. Hemelrijk, D. A. P. Reid, H. Hildenbrandt, and J. T. Padding, Fish Fish. (to be published).

[17] S. D. Kelly and R. M. Murray, Int. J. Robust Nonlinear Control 10, 217 (2000).

[18] S. Alben and M. J. Shelley, Proc. Natl. Acad. Sci. U.S.A. 102, 11163 (2005).

[19] S. Alben, C. Witt, T. V. Baker, E. Anderson, and G. V. Lauder, Phys. Fluids 24, 051901 (2012).

[20] X. J. Zhu, G. W. He, and X. Zhang, Comput. Fluids 97, 1 (2014).

[21] X. J. Zhu, G. W. He, and X. Zhang, J. Fluid Mech. 751, 164 (2014).

[22] W. X. Huang, S. J. Shin, and H. J. Sung, J. Comput. Phys. 226, 2206 (2007).

[23] See Supplemental Material at http://link.aps.org/ supplemental/10.1103/PhysRevLett.113.238105 for (a) details regarding numerical issues, generality of results, instantaneous wake structures, and computation of vortexinduced velocity; (b) five videos corresponding to the wake structures for various parameter values.

[24] J. C. Liao, D. N. Beal, G. V. Lauder, and M. S. Triantafyllou, Science 302, 1566 (2003).

[25] L. B. Jia and X. Z. Yin, Phys. Rev. Lett. 100, 228104 (2008).

[26] C. M. Breder, Jr., Ecology 35, 361 (1954).

[27] R. Godoy-Diana, C. Marais, J. L. Aider, and J. E. Wesfreid, J. Fluid Mech. 622, 23 (2009). 